\title{
Recall of logical and pragmatic implications in sentences with dichotomous and continuous antonyms
}

\author{
WILLIAM F. BREWER and EDWARD H. LICHTENSTEIN \\ University of Illinois at Urbana-Champaign, Champaign, Illinois 61820
}

\begin{abstract}
Thirty-six subjects recalled sentences containing dichotomous or continuous antonyms in affirmative or negative form. The subjects made a considerable number of meaning-preserving recall errors for both dichotomous antonyms (not alive recalled as dead) and continuous antonyms (not hot recalled as cold). The negation of a dichotomous antonym logically implies its antonym, whereas the negation of a continuous antonym only pragmatically implies its antonym. Thus, the results suggest that subjects hearing sentences containing a logical or pragmatic implication tend to make the logical or pragmatic inference involved. Then, in recall, they do not remember that this was an inference and make the error of recalling the logical or pragmatic implication in place of the presented sentence.
\end{abstract}

The purpose of the present experiment is to compare the recall of logical and pragmatic implications in sentences containing dichotomous and continuous antonyms. A sentence logically implies another sentence when the second sentence necessarily follows from the first. Thus, the sentence $I$ regretted that I ate the whole pizza logically implies $I$ ate the whole pizza (cf., Kiparsky \& Kiparsky, 1970). A sentence pragmatically implies another sentence when the information in the first sentence leads the hearer to expect something neither explicitly stated nor necessarily implied by the first sentence. For example, the sentence The karate champion hit the cinder block pragmatically implies The karate champion broke the cinder block. Robin Lakoff (1971) has noted that the conjunction but is frequently used in a sense in which it indicates a "denial of expectation." This observation makes it possible to develop a very useful test for pragmatic implication. If one sentence pragmatically implies another, an acceptable sentence results when the second sentence is negated and conjoined to the first with the conjunction but. For example, The karate champion hit the cinder block but he did not break it is an acceptable sentence.

The distinction between logical implication and pragmatic implication can be applied to the different semantic relations that underlie different antonym pairs. Some antonyms refer to aspects of the world that are dichotomous (e.g., dead-alive, equal-unequal). Since each member of a dichotomous antonym pair refers to one half of a binary distinction, the negation of one logically implies the other (not dead logically implies alive; dead logically implies not alive).

Another class of antonyms refers to aspects of the world that are continuous (e.g., hot-cold, tall-short). These words refer to the opposite ends of a continuum,

We thank Jerome Feldman for assistance in pilot work, Ellen Brewer for assistance with the statistical analysis, and Richard Harris for his comments on the paper.

Requests for reprints should be sent to William F. Brewer, Department of Psychology, Psychology Building, University of Illinois, Champaign, Illinois $\mathbf{6 1 8 2 0}$. but do not exhaust the continuum. The negation of a continuous antonym pragmatically implies its opposite. To say that something is not hot may suggest that it is cold, but does not logically imply that it is, since the term not hot could also be used to refer to something that was warm, neutral, cool, or any point other than hot along the temperature dimension. Affirmative continuous antonyms seem to form an intermediate group. As with the dichotomous antonyms, if the assertion of an affirmative continuous antonym is true, it logically follows that the negation of its opposite is true. However the affirmative continuous antonyms are unlike the dichotomous antonyms (and like the negative continuous antonyms) in that there is an asymmetry in the information contained in the antonym and the negation of its opposite. For example, if something is hot it logically follows that it is not cold, but to say that something is hot is to provide more information than to say that it is not cold. For further discussion of the dichotomous-continuous distinction see the discussion of "contradictory-contrary" in Webster's Dictionary of Synonyms (1951) and the discussion of "complementarity-antonymy" in Lyons (1968).

In addition to these semantic relationships, antonyms also vary in type of surface form. Thus, some dichotomous antonyms are opaque and give no surface morphological indication that they are related (e.g., dead-alive). Other dichotomous antonyms are transparent, with the antonym relation expressed by a prefix attached to a common root (equal-unequal). Continuous antonyms are predominately opaque.

Because of the different types of semantic relationships that exist for antonym pairs, they can be used to test three different hypotheses relating implications and memory: (a) Sentences are remembered in terms of their surface structures, so subjects will recall the original sentences and will not make the error of recalling the logical or pragmatic implications of the original sentence (surface structure hypothesis). (b) Sentences are recalled in terms of their underlying 
and logically implied meanings, so subjects will sometimes make the error of recalling the logical implication of the presented sentence, but will not make the error of recalling the pragmatic implication of the presented sentence (logical implication hypothesis). (c) Sentences are recalled in terms of their pragmatic meanings. The different types of knowledge constructed from sentences (surface structure, logical implications, pragmatic implications) are not well distinguished in memory, so errors in sentence recall may include either the logical implication or pragmatic implication of the original sentence (pragmatic implication hypothesis).

Few experiments have directly tested these hypotheses. However, experiments showing that recognition memory for the meaning of sentences is better than recognition memory for surface structure (Sachs, 1967) and experiments showing that subjects tend to retain the underlying meaning of sentences in recall (Bock \& Brewer, 1974) suggest that the surface structure hypothesis is likely to be false. Harris (1974) has shown that subjects hearing sentences such as Miss America said that she played the tuba tended, in memory, to make truth-value judgments in the direction of the pragmatic implication of the original sentence (e.g., they judged Miss America played the tuba to be true rather than indeterminate). This result tends to support the pragmatic implication hypothesis.

Fillenbaum (1966) has carried out a study of recognition memory for sentences containing dichotomous and continuous antonyms and their negations. The study was designed to examine syntactic theories of sentence memory and was not intended to provide evidence to discriminate between the three hypotheses examined in the present study. The data presented in the article are in the form of the "mean preponderance of meaning.preserving over meaning-changing errors." While these data do not provide a precise test of the surface structure, logical implication, and pragmatic implication hypotheses, the finding that there were a number of confusions between antonyms and their negated opposites can be taken as evidence against the surface structure hypothesis, and the finding that there were more meaning-preserving responses for dichotomous antonyms than for continuous antonyms can be interpreted as favoring the logical implication hypothesis. However, since there was no explicit analysis of whether negated continuous antonyms were confused with their affirmative opposites at a level greater than chance, the study is perhaps better interpreted as not discriminating between the logical implication hypothesis and the pragmatic implication hypothesis.

Thus, the purpose of the present experiment is to compare the surface structure, logical implication, and pragmatic implication hypotheses through the recall of sentences containing dichotomous and continuous antonyms and their negations. The surface structure hypothesis predicts few confusions in recall and no difference between meaning-preserving errors (alive recalled for not dead) and nonmeaning-preserving errors (alive for dead). The logical implication hypothesis predicts confusions in recall for dichotomous antonyms and the negations of their opposites (alive for not dead; not dead for alive) but not for negated continuous antonyms and their affirmative opposites (cold for not hot). The pragmatic implication hypothesis predicts confusions in recall both for dichotomous antonyms and the negation of their opposites and for negated continuous antonyms and their affirmative opposites. Since affirmative continuous antonyms and the negations of their opposites (not hot for cold) form an intermediate case, it is not clear that they can be used to discriminate between the logical implication hypothesis and the pragmatic implication hypothesis.

\section{METHOD}

\section{Subjects}

The subjects were 36 introductory psychology students who participated in partial fulfillment of a course requirement.

\section{Materials}

A pool of about 400 antonym pairs was developed from standard dictionaries of antonyms, word association norms for opposites, and other published works on antonyms. Two raters selected 92 dichotomous antonym pairs and 57 continuous antonym pairs from the original pool. Pairs were classified as dichotomous if the negation of one member of a pair logically implied the other (e.g., not on logically implies off $)$. They were classified as continuous if the negation of one member did not necessarily imply the other (not cold need not imply hot). From the intermediate pool two raters selected a final sample of 20 continuous pairs on the basis of clarity of antonym relation and high frequency of usage; 30 dichotomous pairs were selected using the same criteria plus the requirement that half the items be transparent and half be opaque.

A sentence frame was developed for each of the 50 antonym pairs. By substituting each member of an antonym pair and its negation into a given frame, four sentences were produced from each antonym pair (e.g., The ice cream was hard. The ice cream was soft, The ice cream was not hard. The ice cream was not soft). The experimental items used in this study consisted of the 200 sentences derived from the 50 antonym pairs.

Four master lists were developed such that each list contained one of the four sentences derived from each antonym pair plus 25 affirmative filler sentences. Half of the experimental items on each master list were negations and half were not. Five 15-item experimental lists were developed from each master list. Each experimental list contained four sentences derived from continuous antonyms, three derived from opaque dichotomous antonyms, three derived from transparent dichotomous antonyms, and five filler items. Order of items was consistent across the master lists and experimental lists, so that the sentences derived from a given antonym pair occurred in the same position in each list.

\section{Design}

There were four groups of nine subjects. Each group of subjects received experimental lists derived from one of the four master lists. Thus, a given subject received one of the four 
possible sentences derived from each of the 50 antonym pairs.

\section{Procedure}

The subjects were run in small groups of four to five. They heard five lists of 15 sentences read by the experimenter. After each list they carried out a short digit-memory task requiring immediate written recall of four successive lists of digits. As soon as the digit task was completed, the subjects were given 3 min to write the sentences they had heard. The answer booklet contained recall cues consisting of the initial noun phrase of each sentence, in the same order as the acquisition list.

\section{RESULTS AND DISCUSSION}

The responses were classified as correct if they were written exactly as presented. They were classified as shifts if they were written as presented except for the substitution of the opposite of the presented antonym plus a meaning-preserving addition or deletion of negation (e.g., false recalled as not true; not false recalled as true). Table 1 gives the number of corrects, the number of shifts, and the proportion of shifts to the sum of corrects and shifts for dichotomous transparent, dichotomous opaque, and continuous antonyms for affirmative and negative forms of presentation.

Although occurrence of an appreciable number of shifts in recall in itself suggests that the surface structure hypothesis is not correct, a more specific analysis is possible. The surface structure hypothesis does not consider the meaning of sentences and so does not take account of the fact that some types of recail errors preserve meaning and some do not. The logical implication hypothesis and the pragmatic implication hypothesis do assume recall for an abstract level of meaning and so predict that recall errors will tend to preserve meaning. In order to compare the surface structure hypothesis with the other two hypotheses, a probability analysis was carried out. In order to make a meaning-preserving error, it is necessary to shift to the antonym of the presented word and either drop or add a negation (false recalled as not true; not false recalled as true). Thus, the two implication hypotheses must predict that these two events will tend to occur together, whereas the surface structure hypothesis predicts independence. The overall probability of making an antonym shift in recall was .080 and the overall probability of adding or dropping a negation was .103; if the events occur independently, the expected probability of both events occurring together would be .008 . The obtained probability of both events occurring together was .062 , more than seven times what would be expected by chance. Thus the subjects tend to conserve meaning in their lexical shifts-a finding predicted by the logical and pragmatic implication hypotheses but not by the surface structure hypothesis

The logical implication hypothesis predicts that subjects will make meaning-preserving recall errors on dichotomous antonyms but not on negated continuous antonyms, while the pragmatic implication hypothesis predicts that subjects will make meaning-preserving errors on both types of items. Since the different types of items showed differences both in the number of corrects and the number of shifts, a shift-ratio score was calculated by dividing shifts by the sum of shifts and corrects. This ratio was used because it gives the proportion of meaning-preserving antonym shifts made, given that a meaning-preserving response was made.

As Clark (1973) has pointed out, both subjects and items must be regarded as random effects in an experiment such as this. However, due to the use of the shift-ratio as the response variable, a single analysis of variance with both subjects and items as random effects cannot be performed. Instead, separate analyses were carried out for subjects and for items.

The shift-ratios for each subject for each treatment combination were calculated and a within-subject analysis of variance was carried out, with antonym type (dichotomous transparent, dichotomous opaque, continuous) and affirmative-negative as fixed factors. Only the main effect of antonym type was significant $[F(2,70)=3.95, p<.05]$. A second analysis of variance was carried out using shift-ratios computed for each antonym pair (keeping the affirmative and negative forms of presentation separate). The main effect of antonym type was not significant $[F(2,47)=2.63$, $p>.05]$, and none of the other usual $F$ ratios was significant. Since the main effect for antonym type was not significant for both subjects and items, the conclusion to be drawn from the overall analysis is that there is no reliable difference in the proportion of shifts for the different categories of antonyms. The crucial items for distinguishing between the logical implication hypothesis and the pragmatic implication hypothesis are the continuous antonyms presented in negative form. The logical implication hypothesis predicts that there will be few if any errors of this type, while the pragmatic implication hypothesis predicts that errors of this type will occur. Examination of the data presented in Table 1

Table 1

Number of Corrects, Number of Shifts, and the Shift Ratio for Each Antonym Type

\begin{tabular}{|c|c|c|c|}
\hline Antonym Type & Corrects & Shifts & $\begin{array}{l}\text { Shift } \\
\text { Ratio }\end{array}$ \\
\hline \multicolumn{4}{|c|}{ Dichotomous Transparent } \\
\hline Affirmative & 137 & 27 & .165 \\
\hline Negative & 162 & 19 & .105 \\
\hline \multicolumn{4}{|c|}{ Dichotomous Opaque } \\
\hline Affirmative & 155 & 15 & .088 \\
\hline Negative & 158 & 16 & .092 \\
\hline \multicolumn{4}{|l|}{ Continuous } \\
\hline Affirmative & 241 & 15 & .059 \\
\hline Negative & 221 & 20 & .083 \\
\hline
\end{tabular}

Note-The number of corrects and number of shifts are based on a total possible of 270 for each of the four dichotomous categories and 360 for each of the two contimuous categories. 
shows that the proportion of recall errors in this cell is not strikingly low, so the data tend to support the pragmatic hypothesis.

A probability analysis can also be used to contrast the two hypotheses. The pragmatic implication hypothesis suggests that subjects will sometimes recall pragmatic implications from presented sentences containing continuous antonyms in negated form and thus predicts that subjects will tend to make errors that involve shifting to the antonym of the presented word and dropping the negation (recalling cold for not hot). The logical implication hypothesis predicts that this type of error will only occur by chance. These predictions were examined by calculating the probability of the occurrence of an antonym shift accompanied by the deletion of the negation for the continuous antonyms presented in negated form. The overall prohahility of making an antonym shift for this type of item was .061 , and the overall probability of deleting a negation for this type of item was .122; if the events were independent, the expected probability of both events occurring together would be .007 . The obtained probability of both events occurring together was .055 , more than seven times what would be expected by chance. Thus, as predicted by the pragmatic implication hypothesis, subjects frequently make the error of recalling the pragmatic implication of a presented sentence rather than the presented sentence itself.

The present experiment suggests that when subjects hear a sentence referring to something as not hot, they sometimes make the inference that the thing referred to is cold, then in recall forget that this was an inference they made from the sentence, and thus make the error of recalling the pragmatic implication in place of the original sentence. The results add to the rapidly growing literature (Bransford, Barclay, \& Franks, 1972; Brewer, in press; Harris, 1974; Johnson, Bransford, \& Solomon, 1973) which suggests that subjects are capable of constructing a variety of different types of knowledge from linguistic and nonlinguistic inputs and that the general aspects of the knowledge are well retained, but the origins of a particular bit of knowledge are often lost from memory.

\section{REFERENCES}

Bock, J. K., \& Brewer. W. F. Reconstructive recall in sentences with alternative surface structures. Journal of Experimental Psychology, 1974, 103, 837-843.

Bransford, J. D., Barclay, J. R., \& Franks, J, J. Sentence memory: A constructive versus interpretive approach. Cognitive Psychology, 1972, 3, 193-209.

Brewer, W. F. The problem of meaning and the interrelations of the higher mental processes. In W. B. Weimer and D. S. Palermo (Eds.), Cognition and the symbolic processes. Potomac, Md: Erlbaum, in press.

Clark, H. H. The language-as-fixed-effect fallacy: A critique of language statistics in psychological research. Journal of Verbal Learning and Verbal Behavior, 1973, 12, 335-359.

Fillenbaum, S. Memory for gist: Some relevant variables. Language and Speech, 1966, 9, 217-227.

Harris, R. J. Memory and comprehension of implications and inferences of complex sentences. Journal of Verbal Learning and Verbal Behavior, 1974, 13, 626-637.

Johnson, M. K., Bransford, J. D., \& Solomon, S. K. Memory for tacit implications of sentences. Journal of Experimental Psychology, 1973, 98, 203-205.

Kiparsky, P., \& Kiparsky, C. Fact. In M. Bierwisch and K, E Heidolph (Eds.), Progress in linguistics. The Hague: Mouton, 1970.

Lakoff, R. If's, and's, and but's about conjunction. In C. J Fillmore and D. T. Langendoen (Eds.), Studies in linguistic semantics. New York: Holt, Rinehart and Winston, 1971.

Lyons, J. Introduction to theoretical linguistics. London: Cambridge University Press, 1968.

Sachs, J. S. Recognition memory for syntactic and semantic aspects of connected discourse. Perception \& Psychophysics. $1967,2,437-442$.

Webster's dictionary of synonyms. Springfield, Mass: Merriam, 1951.
(Received for publication July 26, 1974; revision accepted September $7,1974$. ) 